bioRxiv preprint doi: https://doi.org/10.1101/2020.09.06.279869; this version posted September 6, 2020. The copyright holder for this preprint (which was not certified by peer review) is the author/funder, who has granted bioRxiv a license to display the preprint in perpetuity. It is made available under aCC-BY-NC-ND 4.0 International license.

\title{
Detection of Plasmodium vivax in a liver sample of a howler-monkey: one evidence more in favour of the identity between Plasmodium simium and $P$. vivax
}

\author{
Buery, J.C. ${ }^{1,7^{*}}$; Duarte, A.M.R.C. ${ }^{2,3}$; Alencar, F.E.C. ${ }^{1}$; Furieri, C. ${ }^{1}$; Mendes, S.L. ${ }^{4,5}$; Loss, A.C. ${ }^{5}$; Vicente, C.R. ${ }^{1}$; Fux, B. ${ }^{1}$; \\ Rezende, H.R. ${ }^{6}$; Cravo, P.V. ${ }^{7}$, Medeiros, M.M. ${ }^{7}$; Arez, A.P. ${ }^{7}$; Cerutti Jr, C. ${ }^{1}$ \\ ${ }^{1}$ Unidade de Medicina Tropical, Universidade Federal do Espírito Santo, Brazil; ${ }^{2}$ Superintendência de Controle de \\ Endemias ${ }^{3}$ Instituto de Medicina Tropical de São Paulo, Universidade de São Paulo, Brazil; ${ }^{4}$ Laboratório de \\ Primatologia, Universidade Federal do Espírito Santo, Brazil; ${ }^{5}$ Instituto Nacional da Mata Atlântica, Brazil; ${ }^{6}$ Núcleo de \\ Entomologia e Malacologia do Espírito Santo, Brazil; ${ }^{7}$ Global Health and Tropical Medicine, Instituto de Higiene e \\ Medicina Tropical, Universidade NOVA de Lisboa, Portugal.
}

*Corresponding author: julyanabuery@gmail.com

\begin{abstract}
Introduction: The residual malaria of Atlantic Forest systems in Brazil occurs as an endemic disease with low frequency of cases. The chronological and spatial distance among the cases indicate an absence of fitness to the classical malaria cycle. This peculiar condition raised the suspicion of a reservoir, possibly the non-human primates. Simian and human malaria occur at the same places in that region, and there is already evidence of molecular identity between the simian parasites, Plasmodium simium and Plasmodium brasilianum, and the human parasites, Plasmodium vivax and Plasmodium malariae, respectively. Two different SNPs identified in the COX1 region of the Plasmodium vivax/simium of the Atlantic Forest reinforced its characterization as a zoonotic parasite. This finding supported the development of a PCR-RFLP protocol to identify such polymorphisms, and to monitor zoonotic malaria transmission. Methods: In the present work, we tested the above-mentioned PCR-RFLP protocol in unprecedented mosquitoes and simian samples collected in Espírito Santo State, Brazil (ES). Results: The parasite found in the simian sample was $P$. vivax, contrary to what the protocol should indicate. In the mosquito samples, the protocol disclosed both forms of the parasite. Conclusion: This result suggests that the previously published pair of SNPs, and, consequently, the PCR-RFLP protocol, are not able to distinguish the dynamics of Plasmodium spp. circulation in the Atlantic Forest endemic area of ES.
\end{abstract}

\section{INTRODUCTION}

Malaria is the most common parasitic disease worldwide, with 228 million cases and 405,000 deaths reported in 2018 (1). Six different species of Plasmodium are the etiologic agents for this mosquito-borne disease in humans (2). Global efforts aim to reduce the prevalence and mortality of malaria by $90 \%$ by 2030 in comparison to 2015 (212 million cases and 429,000 deaths globally) (3). However, several factors are hampering disease control, such as the presence of asymptomatic human carriers, drug and insecticide resistance, and, in particular cases, the existence of animal reservoirs that are often underestimated. The limited ability to identify asymptomatic carriers impairs malaria control, since these cases are not treated and thus remain as silent reservoirs for transmission. Furthermore, in specific endemic scenarios where non-human primates might participate in the life cycle of the parasite, the risk of ongoing transmission may also increase $(4,5)$.

In the American continent, Brazil is the second country with the highest malaria frequency, with 194,271 cases in 2018, where Plasmodium vivax, Plasmodium falciparum and Plasmodium malariae are the most common etiologic agents. Most of the infections are caused by P. vivax, with the Amazon region comprising $99 \%$ of the reported cases nationwide. The remaining 1\% transmission occurs in the extra-Amazonian Brazilian area. Although transmission in such areas was interrupted in the 1960s, there are still sporadic cases in regions of dense Atlantic Forest, with frequent case reports characterized by a special transmission cycle and clinical presentations. This unusual epidemiological setting has been termed Bromeliad-malaria, because the malariatransmitting mosquitoes breed in the axils of the Bromeliaceae, being relatively frequent in the rural areas of the country's coastal states. In such areas, $P$. vivax is commonly recognized as the etiologic agent of human infections, despite the occasional occurrence of $P$. malariae. 
bioRxiv preprint doi: https://doi.org/10.1101/2020.09.06.279869; this version posted September 6, 2020. The copyright holder for this preprint (which was not certified by peer review) is the author/funder, who has granted bioRxiv a license to display the preprint in perpetuity. It is made available under aCC-BY-NC-ND 4.0 International license.

The Espírito Santo State (ES), located in the south eastern region of Brazil, is a model site to investigate the unusual transmission of Bromeliad-malaria, as it has large fragments of dense Atlantic Forest, and the highest number of cases nationwide. In what concerns the spatial and temporal distances between reported cases in these regions, the transmission cycle does not fit the traditional malaria cycle, as cases in nearby areas occur over a large time span between one and another, separated by several weeks, suggesting the occurrence of a zoonosis, with infected simians participating in the epidemiology. Plasmodium simium and Plasmodium brasilianum are the confirmed etiologic agents of simian infections, despite the studies based on genetic similarities suggesting that they are the same species as $P$. vivax and $P$. malariae, respectively (6-10).

Brasil et al (2017) (11) have previously studied a similar kind of transmission cycle in Rio de Janeiro State (RJ), by sequencing the complete mitochondrial genome (mtDNA) of $P$. vivax/simium recovered from humans and non-human primates. They detected a pair of SNPs in the COX1 gene, which was constant in all the samples tested, but different from sequences obtained previously from the Amazon region (11). While the sequences from RJ comprised the nucleotides $\mathrm{C}$ at position 3535 and $\mathrm{G}$ in the position 3869 those from the Amazon had, respectively, the nucleotides $\mathrm{T}$ and $\mathrm{A}$ at the corresponding positions. As a conclusion, the authors suggested that such SNPs are able to distinguish $P$. simium, classified as the simian parasite causing zoonotic malaria in Atlantic Forest, from P. vivax, the human parasite circulating in Amazon region. Buery et al (2017) (12) also sequenced the mitochondrial $P$. vivax/simium genome from diverse samples collected in an area of preserved Atlantic Forest in ES without migration history to the endemic states of the Amazon region and obtained different conclusions. Opposing the observations in RJ (11), the sequences obtained from Anopheles mosquitoes captured in the inner Atlantic Forest of ES revealed haplotypes consistent with the Amazon P. vivax SNPs pattern (12). Also, there was no common pattern of SNPs that allowed this classification of parasites in at least three samples from humans, meaning that these SNPs were not able to distinguish between the two forms in ES. Both studies importantly documented the presence of the same parasite species in both human and simian hosts. However, such findings are insufficient to confirm a zoonotic cycle.

A subsequent study by de Alvarenga et al (2018) (13) reported a novel PCR-RFLP protocol to detect polymorphisms in the mitochondrial COX1 gene, as previously proposed by Brasil et al (2017) (11). This protocol is directed at identifying $P$. simium in non-human primates and humans from the Atlantic Forest region, aiming to monitor zoonotic malaria transmission in Brazil. This was further used for epidemiological surveys in human and simian samples obtained in other Brazilian studies conducted in RJ $(14,15,16)$. In a bid to test this simple and cost-effective tool in a different geographic area, our group used unprecedent ES samples, whose results we report herein.

\section{METHODS}

In 2016, mosquitoes were collected in Valsugana Velha (VV), a district of Santa Teresa city (ES State), that is largely covered by well-preserved Atlantic Forest and where most of the Bromeliad-malaria takes place (19 57'58.4 "S, 40³4'45.2" W) (4). Anopheles (Kerteszia) cruzii female adults were pooled in groups of 10, which were collected in the same trap on the same date. Liver samples came from simians that perished due to yellow fever epizootics of 2016- 2017 not far from the same region of Atlantic Forest fragments cited above. Sampling of simian and mosquito specimens was approved by the Instituto Brasileiro do Meio Ambiente e dos Recursos Naturais Renováveis/Sistema de Autorização e Informação em Biodiversidade (IBAMA/SISBIO) (licenses numbers 15.191 and 19.227-1, respectively). After DNA extraction, samples were screened for $P$. vivax trough a $18 \mathrm{~S}$ rRNA based qPCR protocol (17). We then followed to characterize these samples according to the previously published protocol by de Alvarenga et al (2018) (13), followed by Sanger sequencing of the amplified amplicon, with minor adaptations.

Targeting the 18S rRNA gene, 29 samples were analysed, of which 16 were positive for $P$. vivax, corresponding to 14 pools of $A$. K. cruzii collected in tree canopies and two livers from monkey bodies. The proposed protocol to differentiate samples between $P$. vivax and $P$. simium (13) were successful for seven samples, six resulting from DNA pools of $A$. $K$. cruzii and one from a simian liver. 
bioRxiv preprint doi: https://doi.org/10.1101/2020.09.06.279869; this version posted September 6, 2020. The copyright holder for this preprint (which was not certified by peer review) is the author/funder, who has granted bioRxiv a license to display the preprint in perpetuity. It is made available under aCC-BY-NC-ND 4.0 International license.

Interestingly, Plasmodium DNA from the simian liver (sample F59) revealed to be $P$. vivax, in contrast to what could be expected in agreement with the previous reports by de Alvarenga et al (2018) (13). Regarding the mosquitoes, both $P$. vivax and $P$. simium were verified (Figure 1).

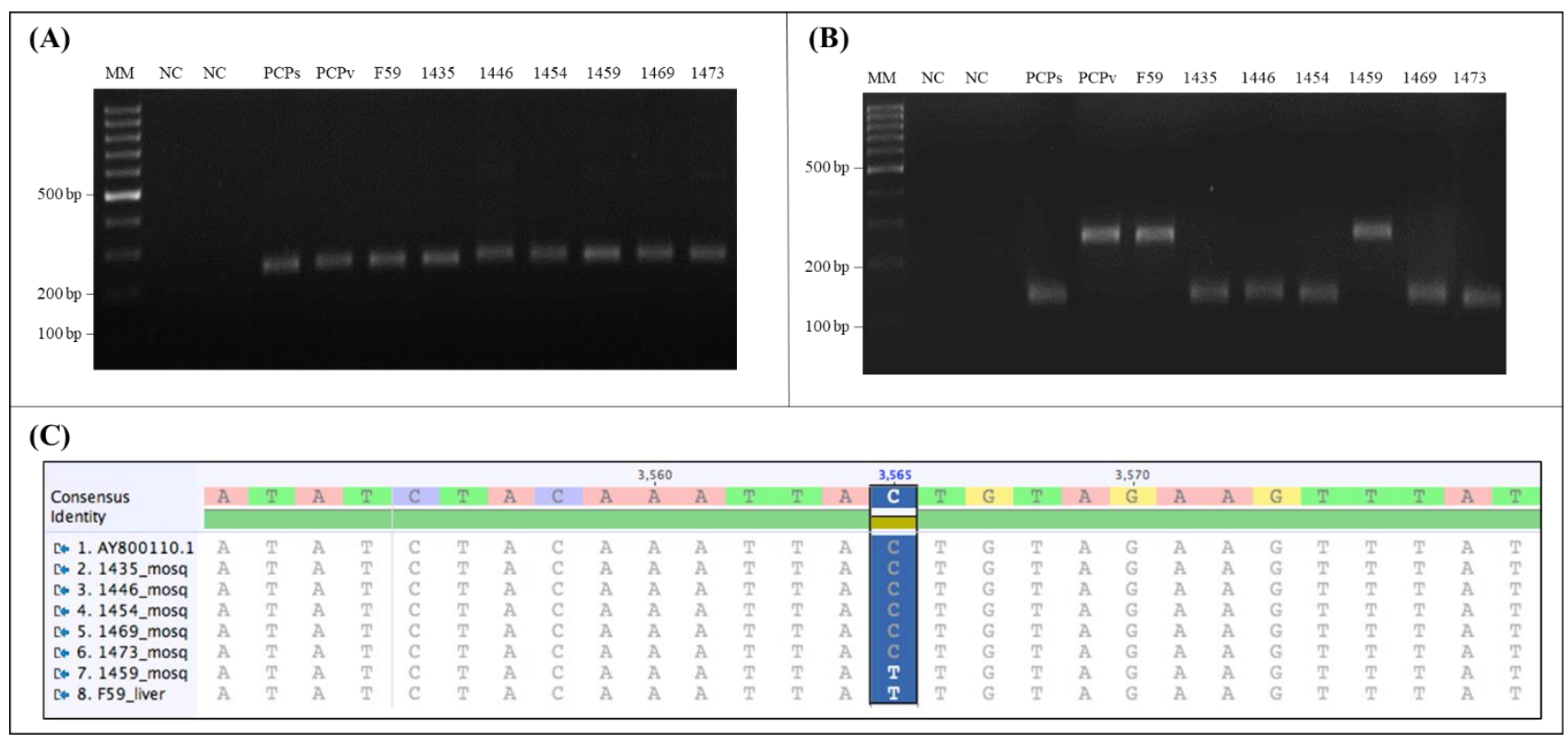

Figure 1 - Proposed protocol of differential diagnosis of $P$. simium infection. (A) Nested/PCR followed by a (B) digestion with HpyCH4III restriction enzyme of one simian liver (F59) and six mosquitoes $(1435,1446,1454,1459,1469,1473)$ samples. 3\% agarose gel stained with GreenSafe. Plasmodium vivax DNA fragments are not digested (244bp), as $P$. simium DNA does, generating fragments of $118 \mathrm{bp}$ and 126bp. MM:1 kb Ladder. NC: Negative Control (no DNA). PCPs: positive control for P. simium, PCPv: Positive Control for P. vivax. (C) Alignment of partial mitochondrial COX1 gene sequence of $P$. simium isolated from free living simian liver (F59) from Atlantic forest; mosquito samples obtained in Atlantic Forest infected with $P$. vivax (1459) and $P$. simium $(1435,1446,1454,1469,1473)$. GenBank sequence from $P$. simium (accession number at GenBank included in the name of sequence). The HpyCH4III restriction site (ACNGT), including SNP T $>\mathrm{C}$ at position 3565, is highlighted.

\section{DISCUSSION}

Lately, in studies performed in the Atlantic Forest region some authors classified all simian samples as $P$. simium under the de Alvarenga et al (2018) protocol indications (11,13-16). Nevertheless, in ES, the parasite found in the simian host was classified as $P$. vivax by the same protocol, raising new issues about the classification of parasites in Atlantic Forest. This result suggests that the previously published SNPs and consequently the protocol to differentiate the parasites are not able to distinguish the dynamics of Plasmodium spp. circulation in the Atlantic Forest. When considering mosquito isolates and the interpretation of the above-mentioned protocol, the findings presented here suggest that both $P$. vivax and $P$. simum are likely to circulate on the canopy, with an equal probability of infecting simian hosts. At this point, the results show an updated scenario in the ES Bromeliad-malaria transmission. In 2017, Buery et al (12) reported a simian isolate from ES with the SNPs proposed by Brasil et al (2017) (11) as a P. simium based on the mtDNA sequence. Plus, mosquito isolates were analysed and none of them presented the nucleotides indicating the simian parasites. Recently, one sample of Plasmodium spp. DNA obtained from simian blood collected in southern Brazil was analysed by the same protocol and revealed the genetic pattern of $P$. vivax presented in the simian sample here reported (14). Unexpectedly, the authors designated the parasite as a non-Plasmodium simium (NPs, animal 4). Herein, the parasite disclosing this pattern was identical to $P$. vivax after qPCR, nested PCR, and Sanger sequencing. Taken together, these pieces of evidence reveal the genetic diversity in this heterogeneous and unusual cycle of transmission, and that only two SNPs do not seem to be sufficient to disclose the real epidemiological situation at Atlantic Forest region.

Improved understanding of the Bromeliad-malaria transmission cycle would strengthen the capability of Health authorities to promote adequate control strategies and would provide useful information to other Public Health 
authorities dealing with similar ecologic conditions around the world. The lack of a comprehensive understanding of the dynamics of the parasite's transmission cycle in the Atlantic Forest is a consequence of a low number of both mammalian and vector samples which are analysed simultaneously but that are often collected in different periods. Accurate characterization of the distinctive genetic features of Plasmodium variants involved in Bromeliad-malaria requires the evaluation of all the putative elements of the cycle obtained from a cross-sectional survey or in a shorter timeframe. To verify a zoonotic cycle, one would have to analyse parasite genetic diversity and estimate the divergence time from the most recent common ancestor by molecular clock analysis. Such an approach was performed to analyse the transmission of $P$. knowlesi, uncovering a higher number of genotypes per infection in simians than in humans (18), which allowed the first unequivocal confirmation of zoonotic transmission of malaria in Southeast Asia (18). Similarly, natural transmission of Plasmodium cynomolgi between simians and humans became known in 2011 in Malaysia (19). Divergence time from the most recent common ancestor based on the analysis of mtDNA revealed the derivation of the species from an ancestral parasite population that existed before human settlement in Southeast Asia. Both findings were able to support the hypothesis of an actual zoonosis, pointing to a recent transfer of the parasites to the humans. In the case of $P$. vivax/simium, however, the evidence points in the opposite direction. The haplotype diversity is lower among the simians, and the phylogenetic analyses indicate a recent transfer of the species from humans to simians $(12,20)$.

\section{CONCLUSION}

The results of this work suggest that the published SNPs and the protocol of differential diagnosis of $P$. simium infection are not able to elucidate the dynamics of Plasmodium spp. circulation in the Atlantic Forest of ES. The evidence until now is not sufficient to determine how and if the transfer of the parasites occurs in the Atlantic Forest, precluding any definite conclusion regarding a zoonotic cycle. Further studies with larger numbers of samples from humans, simians, and vectors, collected over a short timeframe will allow deeper understanding of the transmission cycle of this singular endemic disease.

Funding: This work was supported by Fundação de Amparo à Pesquisa e Inovação do Espírito Santo (FAPES) [grant number 344/2018] and Fundação de Amparo à Pesquisa do Estado de São Paulo (FAPESP) [grant number 2014/10919-4]. JCB has a FAPES fellowship [grant number PROFIX10/2018]. ACL has a CNPq fellowship [grant number 302375/2020-1]. Our acknowledgments to Fundação para a Ciência e Tecnologia (FCT) for funds to GHTM [grant number UID/04413/2020].

Author contributions: Buery, J.C.: Conceptualization, Validation, Investigation, Writing - original draft, Project administration, Funding acquisition. Duarte, A.M.R.C.: Resources, Investigation, Writing - Review \& Editing. Alencar, F.E.C. and Vicente, C.R.: Writing - Review \& Editing, Visualization. Furieri, C.; Mendes, S.L.; Rezende, H.R. and Fux, B.: Resources, Data Curation.Loss, A.C.: Formal analysis, Writing - Review \& Editing. Cravo, P.V. and Medeiros, M.M.: Validation, Writing - Review \& Editing. Arez, A.P.: Validation, Resources, Writing - Review \& Editing, Supervision. Cerutti Jr, C.: Conceptualization, Resources, Writing original draft, Project administration, Supervision.

\section{REFERENCES}

1 World Health Organization. World malaria report. 2019. 232p.

2 Saralamba N, Nosten F, Sutherland CJ, et al. Genetic dissociation of three antigenic genes in Plasmodium ovale curtisi and Plasmodium ovale wallikeri. PLoS One. 2019;14(6):e0217795. doi:10.1371/journal.pone.0217795.

3 World Health Organization. World malaria report. 2016. 148 p.

4 Cerutti C Jr, Boulos M, Coutinho AF, Hatab C, Rezende HR, Duarte AM, et al. Epidemiologic aspects of the malaria transmission cycle in an area of very low incidence in Brazil. Malar J. 2007; 6:33. 
bioRxiv preprint doi: https://doi.org/10.1101/2020.09.06.279869; this version posted September 6, 2020. The copyright holder for this preprint (which was not certified by peer review) is the author/funder, who has granted bioRxiv a license to display the preprint in perpetuity. It is made available under aCC-BY-NC-ND 4.0 International license.

5 Duarte AM, Malafronte RS, Cerutti C, Curado I, Paiva BR, Maeda AY, et al. Natural Plasmodium infections in Brazilian wild monkeys: reservoirs for human infections? Acta Trop. 2008; 107:179-85.

6 Goldman IF, Qari SH, Millet PG, Collins WE, Lal AA. Circumsporozoite protein gene of Plasmodium simium, a Plasmodium vivax-like monkey malaria parasite. Mol Biochem Parasit. 1993; 57:177-80.

7 Escalante AA, Freeland DE, Collins WE, Lal AA. The evolution of primate malaria parasites based on the gene encoding cytochrome b from the linear mitochondrial genome. Proc Natl Acad Sci USA. 1998; 95:8124 9.

8 Leclerc MC, Durand P, Gauthier C, Patot S, Billotte N, et al. Meager genetic variability of the human malaria agent Plasmodium vivax. Proc Natl Acad Sci USA. 2004; 101:14455-60.

9 Lim CS, Tazi L, Ayala FJ. Plasmodium vivax: recent world expansion and genetic identity to Plasmodium simium. Proc Natl Acad Sci USA. 2005; 102:15523-8.

10 Tazi L, Ayala FJ. Unresolved direction of host transfer of Plasmodium vivax v. P. simium and P. malariae v. P. brasilianum. Infect Genet Evol. 2011; 11:209-21.

11 Brasil P, Zalis MG, Pina-Costa A, Siqueira AM, Bianco C Jr, Silva S, et al. Plasmodium simium causing human malaria: a zoonoses with outbreak potential in the Rio de Janeiro Brazilian Atlantic forest. Lancet Glob Health. 2017; 5:1038-46.

12 Buery JC, Rodrigues PT, Natal L, et al. Mitochondrial genome of Plasmodium vivax/simium detected in an endemic region for malaria in the Atlantic Forest of Espírito Santo state, Brazil: do mosquitoes, simians and humans harbour the same parasite? Malar J. 2017;16(1):437. doi:10.1186/s12936-017-2080-9.

13 de Alvarenga DAM, Culleton R, de Pina-Costa A, et al. An assay for the identification of Plasmodium simium infection for diagnosis of zoonotic malaria in the Brazilian Atlantic Forest [published correction appears in Sci Rep. 2019 Nov 21;9(1):17521]. Sci Rep. 2018;8(1):86.

14 Nunes AJD, Alvarenga DAM, de Souza Junior JC, et al. Plasmodium infection and its association with biochemical and haematological parameters in free-living Alouatta guariba clamitans (Cabrera, 1940) (Primates: Atelidae) in Southern Brazil. Mem Inst Oswaldo Cruz. 2020;114:e190210.

15 Abreu FVS, Santos ED, Mello ARL, et al. Howler monkeys are the reservoir of malarial parasites causing zoonotic infections in the Atlantic forest of Rio de Janeiro. PLoS Negl Trop Dis. 2019;13(12):e0007906.

16 de Abreu FVS, Gomes LR, Mello ARL, et al. Frozen blood clots can be used for the diagnosis of distinct Plasmodium species in man and non-human primates from the Brazilian Atlantic Forest [published correction appears in Malar J. 2019 May 14;18(1):168]. Malar J. 2018;17(1):338.

17 Rubio JM, Benito A, Roche J, Berzosa PJ, García ML, Micó M, et al. Seminested, multiplex polymerase chain reaction for detection of human malaria parasites and evidence of Plasmodium vivax infection in Equatorial Guinea. Am J Trop Med Hyg. 1999; 60:183-7.

18 Lee KS, Divis PCS, Zakaria SK, Matusop A, Julin RA, Conway DJ, et al. Plasmodium knowlesi: reservoir hosts and tracking the emergence in humans and macaques. PLoS Pathog. 2011;7:1002015.

19 Ta TH, Hisam S, Lanza M, Jiram AI, Ismail N, Rubio JM. First case of a naturally acquired human infection with Plasmodium cynomolgi. Malar J. 2014;13:68. doi:10.1186/1475-2875-13-68.

20 Rodrigues PT, Valdivia HO, Oliveira TC, Alves JMP, Duarte AMRC, Cerutti Jr C, et al. Human migration and the spread of malaria parasites to the New World. Sci Rep. 2018; 8(1):1993. 
